\title{
Essais
}

Revue interdisciplinaire d'Humanités

Hors-série 1 | 2013

L'estrangement

\section{Le distant et le familier. Giorgio del Giglio «Pannilini » et son lecteur}

\section{Florence Buttay}

\section{OpenEdition}

1 Journals

Édition électronique

URL : http://journals.openedition.org/essais/2362

DOI : 10.4000/essais. 2362

ISSN : 2276-0970

Éditeur

École doctorale Montaigne Humanités

\section{Édition imprimée}

Date de publication : 15 octobre 2013

Pagination : 147-159

ISBN : 978-2-9544269-7-4

ISSN : 2417-4211

\section{Référence électronique}

Florence Buttay, «Le distant et le familier. Giorgio del Giglio «Pannilini » et son lecteur », Essais [En ligne], Hors-série 1 | 2013, mis en ligne le 05 octobre 2020, consulté le 18 octobre 2020. URL : http:// journals.openedition.org/essais/2362 ; DOI : https://doi.org/10.4000/essais.2362 


\section{Le distant et le familier. Giorgio del Giglio « Pannilini » et son lecteur}

\section{Florence Buttay}

La lecture des écrits d'un voyageur du XVI ${ }^{\mathrm{e}}$ siècle implique une double distance : la distance temporelle entre le voyageur et nous, qui le lisons aujourd'hui, et la distance, géographique et donc aussi culturelle, dont le voyageur rendait compte pour ses lecteurs contemporains. Dans le cas du voyageur, bien oublié, qui fait l'objet de ces pages, Giorgio del Giglio "Pannilini » (1507 ?-1579 ?), cette distance géographique n'est pas le seul écart qui le séparait de ses lecteurs. D’origine incertaine, probablement issu d'une famille marrane exilée en Italie à la fin du $\mathrm{XV}^{\mathrm{e}}$ siècle, Giorgio a exercé de multiples activités, la plupart liées au renseignement, et navigué aux marges de la Méditerranée chrétienne, tantôt côté turc, tantôt côté chrétien et, de ce côté-ci, tantôt au service de Florence, tantôt au service du pape, tantôt au service de l'Espagne. Il a embrassé par deux fois la religion musulmane et est revenu par deux fois dans le giron de l'Église, non sans être tenté par le calvinisme. Son nom varie au cours des années 1550-1560 : il signe Giorgio del Giglio, puis Pannilini, puis De Franceschi Pannilini, usurpant des noms de famille siennois respectables. Lécart de Giorgio del Giglio par rapport à ses contemporains de la péninsule italienne est à la fois son problème et le moyen qu'il a choisi pour le résoudre. Le but de l'écriture de ses volumineux manuscrits est en effet, on le verra, de réduire sa propre étrangeté à la société chrétienne dans laquelle il cherche une place et, en même temps, seule cette étrangeté, seule sa connaissance intime du monde turc, sa "perizia nelle cose de' Turchi ", grâce à ses séjours prolongés au milieu d'eux et à son identité trouble de renégat, lui assurent l'oreille de protecteurs et donc les moyens de vivre dans cette société italienne de la deuxième moitié du XVI ${ }^{\mathrm{e}}$ siècle. D'où une tension constante dans son écriture entre le distant et le familier. Le distant est ramené au familier ou doit permettre de rêver une transformation du familier. L'« estrangement » est ainsi une notion très pertinente pour appréhender la distance de Giorgio à ceux qu'il voudrait ses compatriotes. Mais elle est tout aussi utile à l'historien pour appréhender les textes que Giorgio produit ; des textes qui, au premier 
abord, relèvent largement de l'illisible. Or, si un texte est incompréhensible, ou illisible ou écrasant d'ennui alors qu'il suscitait la curiosité, alors quelque chose a bougé dans les 500 ans qui nous en séparent, qui nous le rend illisible. C'est cet écart même qui devient objet d'enquête.

\section{Giorgio del Giglio et son œuvre}

L'enquête entreprise autour de Giorgio peut paraître étrange : personnage mineur, petite main des réseaux diplomatiques tissés entre l'Italie et l'Orient méditerranéen, il colporte des informations médiocres. Il suscite à juste titre la méfiance de nombre de ses interlocuteurs. L'un d'eux, autre informateur au service de Côme de Médicis, le traite de " mariuolo fallito " ${ }^{1}$.

Mais il a consacré beaucoup d'énergie à la rédaction d'ouvrages volumineux. Deux gros manuscrits témoignent de cet effort. Conservés respectivement au Vatican (fonds Barberini latini) et à Sienne (Bibliothèque degli Intronati), ils sont intitulés Viaggi ou Il Gran Viaggio, mais ils tiennent autant du livre de famille, de ricordanze, de l'atlas, du récit de conversion... Ils ont été rédigés entre 1564 (version perdue) et 1580 environ, quand, après deux conversions à l'Islam, en 1550 et 1560, il revient en Italie et en chrétienté apparemment définitivement ${ }^{2}$. Les volumes conservés ne sont sans doute pas les seuls que l'auteur ait produits. En effet, Giorgio semble avoir été atteint d'un prurit d'écriture de soi. Il n'a de cesse de reproduire et de retravailler le récit de sa vie, variant sans cesse la chronologie, les faits, les personnages. On le voit aussi bien dans ces manuscrits que dans les lettres qu'il a écrites aux puissants pour vendre ses services et qui sont parvenues jusqu'à nous (adressées au grand duc de Toscane, à la République de Gênes, au vice-roi de Naples, etc.) $)^{3}$.

Les deux manuscrits conservés sont certes très proches dans la trame générale du contenu, mais le manuscrit de Sienne fournit de nombreuses informations divergentes ou complémentaires par rapport au manuscrit Barberini. Plus que deux versions, ce sont deux livres traitant des mêmes objets. La version siennoise a d'évidence été commencée bien après ce dernier. En effet, le Barb. Lat. 4791 s'intitule Viaggio comenciato damme Giorgio Gilii Pnnolini della cicta

1 Archivio di Stato Firenze, Mediceo del Principato, 484, fol. 117, lettre du bailo Albertaccio degli Alberti, de Pera, $1^{\text {er }}$ avril 1560.

2 Biblioteca Apostolica Vaticana, fonds Barberini latini, ms. 4791 et Biblioteca Comunale di Siena, ms. L. IV, 39. Sur tout cela, voir Florence Buttay, «Les captivités de Giorgio del Giglio Pannilini ", in François Moureau (éd.), Captifs en Méditerranée (XVIe-XVIII siècles), Paris, PUPS, 2007, p. 59-76.

3 Florence Buttay, "Lettres d'un imposteur. La construction épistolaire d'une identité : Giorgio del Giglio Pannilini (v. 1507-v. 1580) », in Jean Boutier, Sandro Landi, Olivier Rouchon (éd.), La Politique par correspondance : les usages politiques de la lettre en Italie, XIV-XVIII siècle, Rennes, PUR, 2009, p. 65-86. 
di Siena per Asia Africa Europa per mare et per terra comenciato ellanno del nostro Signore 1542 fino illanno nel 1564, tandis que le manuscrit de Sienne donne le terminus de $15799^{4}$. Pourtant Pannilini continue l'écriture du manuscrit romain bien au-delà et la dernière mention est celle de la naissance de son fils Gian Paolo, le 10 janvier 1579, justement ${ }^{5}$. Peut-être commence-til à copier le manuscrit des Intronati quand il estime à peu près achevé le manuscrit romain, même si aucun des deux ouvrages n'est véritablement clos. Cependant les Voyages de Sienne ne contiennent pas ces annotations journalières et personnelles qui rapprochent le manuscrit romain d'un livre de ricordanze (naissance des enfants, comptes, recettes...). Il semble être une mise au propre pour une circulation voire une possible publication, ainsi que le suggèrent la mise en place et la numérotation des cahiers. On remarque aussi la mention " copiato " répétée entre les fol. 1 à 6 qui conduit à envisager une autre copie, dont le manuscrit des Intronati serait le prototype. Les Voyages de Pannilini ont en effet connu une circulation manuscrite, quelques érudits toscans du XVII e siècle citent les voyages de "Pancrolini " ultime variation sur son nom, issu soit d'une mauvaise lecture de Pannolini soit d'un ultime masque de l'auteur dont on n'aurait pas d'autre trace.

On peut distinguer deux temps dans la rédaction du manuscrit vatican, qui est donc la version la plus ancienne de l'ouvrage qui nous est parvenue, sachant que cette rédaction s'est étendue, d'après les indications contenues dans le texte, sur une quinzaine d'années.

1. Le projet initial (fol. 1-154v) correspond au titre : il s'agit du récit de la vie de l'auteur de sa naissance à son reniement (1545-1550) suivi de tous les voyages effectués au service du Grand Turc jusqu'en 1559 ; enfin le retour, en deux fois, à la Chrétienté (1559-1565 ou 1567). Les dates données par Giorgio del Giglio sont souvent contradictoires.

2. Par la suite (à partir du fol. 155) : l'auteur cherche à la fois à rendre compte des connaissances accumulées pendant ces années de voyages et à poursuivre le récit de sa vie. Il consacre en particulier plus de 100 feuillets à un Isolario (fol. 176v-287), description de toutes les îles de toutes les mers de l'Ancien et du Nouveau Monde. Mais on trouve aussi une histoire et description de la cour du Grand Turc, des planches de botanique. Enfin l'auteur raconte sa participation à l'expédition avec Don Juan d'Autriche, au début des années 1570.

Le manuscrit du Vatican, comme celui de Sienne, est entièrement de la même main, agrémenté de nombreux dessins à la plume, quelques fois rehaussés de couleurs : plans de villes, plantes, navires, paysages... (illustration 1)

4 BAV, Barb. lat. 4791, fol. [IV]r. Sienne, BC, ms. L. IV. 39, fol. 1r : Il gran viagio comenciato da me Giorgio de Francieschi Pannilini della citta di Siena per Asia e Africa Europa per mare et per terra comenciando nellanno del nostro Signore 1507 in fino nel 1579. 


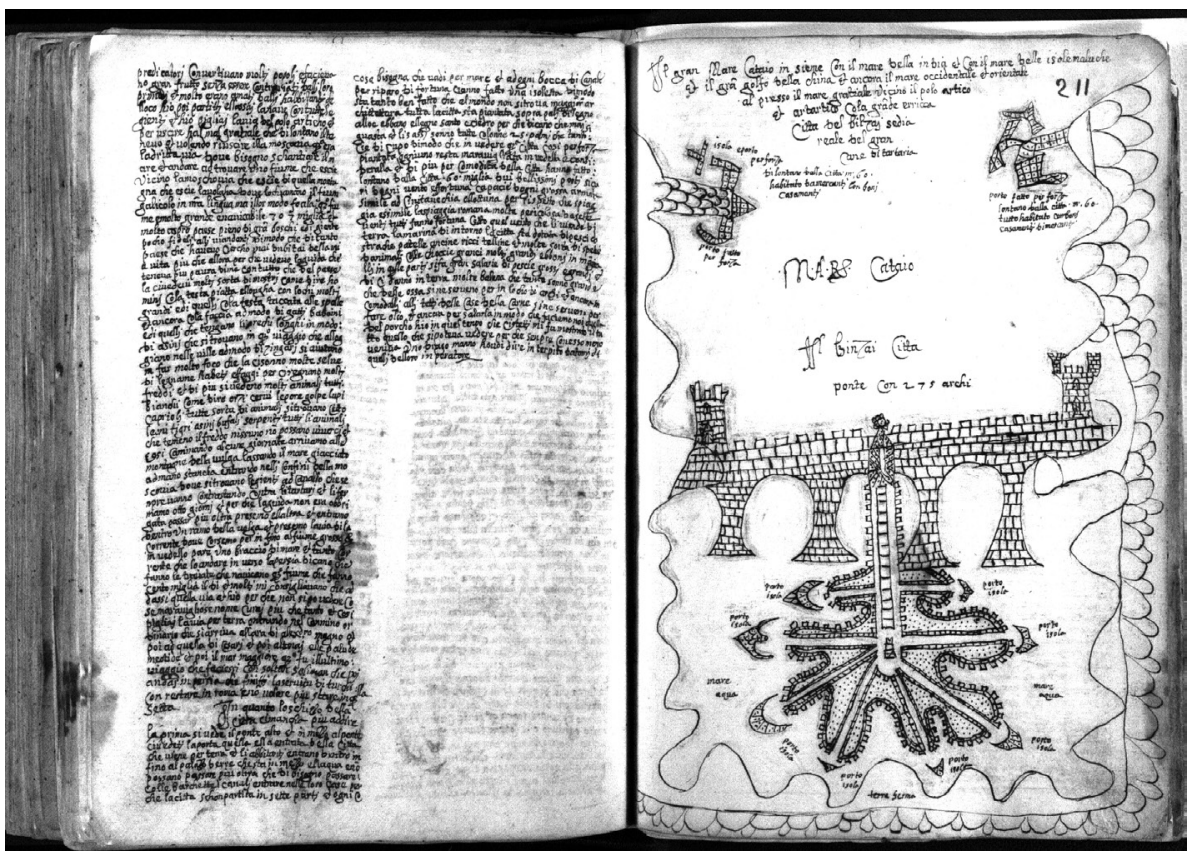

On remarque que, bien qu'il y ait plusieurs temps de rédaction, le projet se répète sur le schéma suivant : un développement biographique est suivi d'un déploiement de connaissances. La vie et les tribulations de Giorgio del Giglio jusqu'à son reniement sont suivies de "voyages " qui donnent lieu à tout une série d'informations géographiques, botaniques, historiques... Le récit du retour en chrétienté constitue une nouvelle tranche autobiographique, à nouveau suivie d'une description du monde (l'Isolario) et d'autres informations historico-géographiques. Enfin, une dernière séquence fait dialoguer deux récits autobiographiques : visions astrologiques/voyage avec les troupes de Don Juan d'Autriche 1570-1573 - récit soi-disant autobiographique d'un concile des religions. Malgré les errements, et en comparant avec le manuscrit de Sienne, ce projet liant étroitement autobiographie, voyages et savoirs se précise : le voyage sert un projet de somme du savoir qui est un projet de description du monde.

Ces manuscrits constituent ainsi des documents fascinants où Giorgio se montre très disert sur la comparaison et la concorde des religions, ou encore la supériorité de la foi des renégats réconciliés, à une date où ce genre de sujets peut vous coûter la vie. Mais c'est aussi un grand dissimulateur, affabulateur, usurpateur (il se fait appeler Pannilini à partir de 1564, avant de préférer se dire De Franceschi Pannilini). On peut mesurer l'écart entre ce qu'il raconte et ce qu'il a fait en retrouvant sa trace dans les archives italiennes. En particulier pour les années 1560 peuvent être reconstituées grâce aux correspondances conservées aux archives d'État de Florence. 


\section{Qu'est-ce que l'historien peut faire de ce genre de textes ?}

Quand j'ai commencé à travailler sur ce personnage, j'ai cherché la collaboration de spécialistes, ottomanistes notamment, et souvent (pas toujours heureusement) ils sont restés sceptiques : Giorgio n'est pas un personnage représentatif, il est un escroc et un affabulateur, on ne sait presque rien de lui, ce qu'il raconte n'est pas original, ses connaissances sont loin d'être en pointe, il n'est pas une source intéressante, fiable, etc. Ces livres peuvent en effet ressembler aux soliloques d'un petit bonhomme marginal et divagant et l'on peut se demander s'ils constituent un objet d'étude sérieux. De ce point de vue, la notion d'" exceptionnel normal » développée par Carlo Ginzburg a été précieuse pour ce travail. Réfléchissant sur l'" exceptionnel-normal » Menocchio, vingt ans plus tard, à la demande de Denis Thouars, il défend le parti de l'étude de cas étranges : «Je dois dire que l'idée que qu'on ne pourrait généraliser qu’à partir de cas moyens, de cas normaux (ou du moins considérés comme tels) ne me parait pas seulement paresseuse : elle me semble insensée [...]. La notion d'“exceptionnel normal" - selon l'oxymoron particulièrement efficace proposé par Edoardo Grendi souligne la possibilité qu'un document rare, du point de vue statistique, "exceptionnel" donc, puisse éclairer un phénomène social diffusé et "normal" " Carlo Ginzburg explique ensuite la méthode qu'il cherche à appliquer à de tels cas, afin d'embrasser une réalité sociale complexe.

Aujourd'hui je proposerais de considérer un individu comme le point d'in-
tersection d'une série d'ensembles différents qui ont chacun des dimensions
variables. Un individu appartient à une espèce (homo sapiens sapiens), à
un genre sexuel, à une communauté linguistique, politique, professionnelle
et ainsi de suite. Parmi ces ensembles il y a aussi celui qui se fonde sur les
empreintes digitales et qui comprend un seul individu. Mais identifier un
individu par ses seules empreintes digitales n'est licite que dans une optique
policière. L'historien doit partir de l'hypothèse que chez tout individu quel
qu'il soit, et même le plus anormal (et presque tout individu l'est-il, ou du
moins peut-il apparaître tel) coexistent des éléments plus ou moins générali-
sables. L'anomalie sera le résultat des réactions réciproques de tous entre tous
ces éléments. Ainsi, parler d'anomalie de manière absolue n'a aucun sens.

Considérer un homme comme un point d'intersection, comme un carrefour qui permette de voir loin dans plusieurs directions, c'est ce que j'aimerais faire de l'étude entreprise autour de Giorgio del Giglio, un homme à la charnière entre différentes manières de voir et de parler du monde.

Je travaille ainsi dans deux directions : d'une part, un marginal comme Giorgio del Giglio Pannilini, est engagé dans un processus permanent de construction de son identité (pour lui et les autres). Construction par l'écri-

6 In Denis Thouard (éd.), Linterprétation des indices. Enquête sur le paradigme indiciaire avec Carlo Ginzburg, Lille, Presses universitaires du Septentrion, 2007, p. 42-43. 
ture, c'est ce dont il nous reste des traces, mais aussi, étroitement lié, en son temps, par la parole. Il s'agit donc d'une enquête sur la manière dont un homme socialement mal intégré se présente, se représente, se fait connaitre. C'est une enquête aussi sur la manière dont, à cinq siècles de distance, l'historien peut à son tour tenter de l'identifier, de le replacer comme un morceau de puzzle dans le paysage politique, culturel, religieux de l'Italie de son temps et jusqu'où il peut le faire. D'autre part, ce travail cherche à reconstituer la culture d'un tel homme. Ce voyageur obscur déroute à la fois par son italien confus et ses récits où merveilleux et affabulation occupent une place importante, plus importante que dans les récits de voyageurs contemporains sur lesquels les spécialistes se penchent d'habitude. C'est ce qui suscite le mépris souvent des rares personnes qui s'aventurent du côté des livres de Giorgio del Giglio. Pourtant, le caractère périmé ou fabuleux des savoirs (géographiques, botaniques, historiques) qu'il véhicule est passionnant pour qui s'intéresse à la circulation des savoirs en Méditerranée. Ces manuscrits témoignent des connaissances accessibles à un " idiota », "illiterato » (c'est-à-dire qui maitrise la lecture et l'écriture du vernaculaire mais reste en dehors de la sphère du latin) et qui vit "amphibie " (pour reprendre le mot de Natalie Zemon-Davis au sujet Léon l'Africain) d'une rive à l'autre de la Méditerranée, de l'Islam au christianisme, de l'économie légale à la contrebande, de la diplomatie officielle à l'espionnage.

De ces directions d'enquête découle le parti-pris de ne pas masquer le travail de l'historien, les interrogations posées, résolues ou non, les fausses pistes, etc., parce que de tels documents invitent à une réflexion sur le sens et la valeur des textes. La démarche anthropologique est à cet égard un modèle. Ainsi, l'enquête passionnante de Monder Kilani, sur les fonctions de l'écrit dans la société d'une oasis tunisienne est exemplaire'. Il ne s'agit pas d'une monographie classique. L'auteur a l'ambition de décrire une société oasienne sans gommer le cheminement par lequel il a établi sa relation avec ce «terrain et a construit la compréhension des objets sociaux et culturels qu'il y découvrait ». Il ne laissera pas, du travail qui l'a conduit, que la figure lisse du narrateur scientifique, " transfiguré en autorité neutre autant qu’omniprésente ».

L'aurais-je voulu sous cette forme que cet ouvrage aurait manqué son but qui est de saisir le mouvement propre à toute construction de connaissance, connaissance de l'anthropologue bien sûr, mais aussi connaissance de l'oasien qui construit son identité, la négocie et la légitime dans un contexte d'interactions multiples. Un tel souci, à l'évidence, ne s'accommode pas d'un texte monologique, fermé sur lui-même et coupé de ses conditions de production ${ }^{8}$.

Dans l'étude des écrits de Giorgio del Giglio également, l'objectif est de reconstituer une construction de connaissance par l'écrit destinée à produire et à défendre une identité.

7 La construction de la mémoire. Le lignage et la sainteté dans l'oasis d'El Ksar, 1992.

8 Ibid., p. 14. 
Monder Kilani montre comment son sens historien du document est bousculé par l'usage qu'en font les habitants de l'oasis d'El Ksar et il rapporte les impasses dans lesquelles il s'est trouvé'. L'enjeu n'est pas seulement l'honnêteté (qui ne doit pas se "transfigurer en autorité neutre autant qu'omniprésente »); il s'agit aussi mais aussi de pouvoir suivre les choix opérés pour aborder ces documents. D'autres choix possibles étaient possibles, sans que leur exposition invalide le discours scientifique, mais au contraire permette au lecteur de juger de sa pertinence. Cela permet aussi de montrer avec quels a priori un chercheur issu d'une certaine tradition académique aborde un texte, une œuvre d'art, etc. Intégrer cela au processus d'écriture, c'est se donner une chance de pouvoir penser contre soi : traquer son objet, en histoire, passe aussi par une lutte contre soi, avec son outillage intellectuel, contre ses automatismes, contre le formalisme, le conformisme introduit par le fait même de devoir respecter une forme d'écriture académiquement acceptable.

Le travail autour des dessins de Giorgio mérite d'être racontée, à cet égard. Ils m'ont beaucoup frappés par leur aspect oriental, sans que je sois évidemment en aucun cas spécialiste et capable de les inscrire dans une tradition graphique particulière (illustration 2). En même temps, la technique employée est tout à fait italienne (Giorgio dessine à la plume sur une esquisse à la sanguine) et dans cette vision, on retrouve des éléments iconographiques bien connus. J'ai présenté ces dessins à plusieurs spécialistes de l'art ottoman qui ont cherché avec moi de quel Orient ils relevaient. Ils n'identifiaient rien, pas d'influence précise mais confirmaient leur origine ou leur influence orientale. Et puis, un jour, en feuilletant des herbiers italiens, je me suis proposé de penser contre mon premier mouvement : étaient-ils si orientaux ou orientalisants ? L'Orient que j'y voyais (dans la forme des yeux ou des montagnes, par exemple, dans l'ornementation des bordures et le traitement ornemental des surfaces (murs, coques des bateaux, etc.) relevait peut-être d'un code de représentation qui m’appartenait. L'enquête n'est pas finie...

9 «Voici une société, qui depuis toujours connaît l'écriture, où le prestige de la culture savante est grand et où la plupart de ses membres savent lire et écrire et qui, pourtant, développe un rapport particulier au document, au point de faire vaciller la frontière que l'on pose habituellement entre le registre de l'oral et celui de l'écrit. L'usage qui est fait ici du document écrit participe d'un mode d'action qui déplace la fonction habituelle de l'écrit comme inscription d'un contenu, d'un sens vers une fonction purement illocutoire. Ce qui compte le plus dans la stratégie rhétorique des oasiens, c'est moins la désignation du contenu du document que l'acte même qui le désigne. C'est l'acte de langage qui compte (...). Chez les oasiens, le discours construit le document et s'y substitue dans le cadre d'une stratégie de la croyance et de la conviction". 


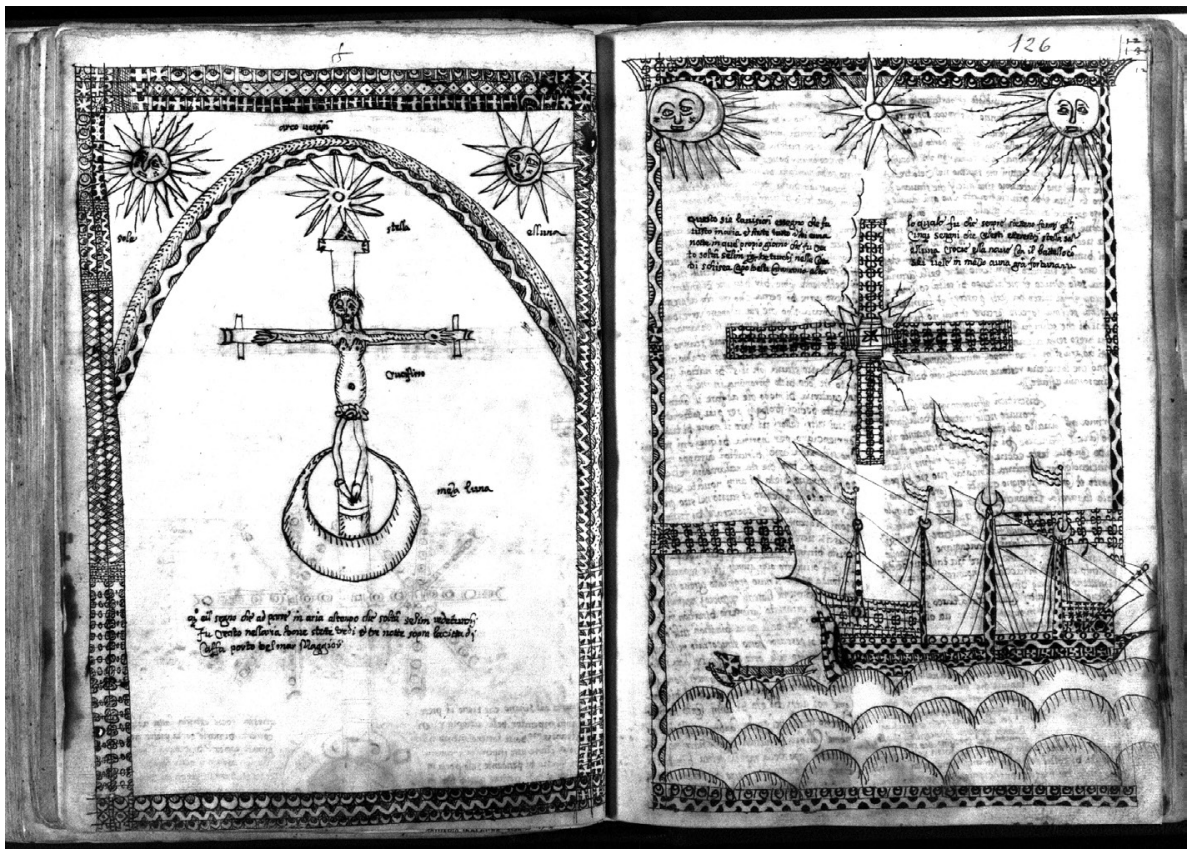

\section{La distance entre Giorgio et son lecteur et ses astuces pour la réduire}

Giorgio est un grand conteur voire un bonimenteur, le lecteur est donc très présent. Le lecteur ou plutôt les lecteurs, car il s'adresse à "voi lettori " ou " lectori ", rarement au singulier. Giorgio l'interpelle, lui demande d'attendre, crée du suspens, s'inquiète qu'il s'ennuie, répète qu'il veut et va tout lui dire sans rien omettre... Par exemple, il raconte ainsi comment il passe de l'Afrique du Nord en Égypte en traversant le désert : " Je ne veux pas manquer de vous dire, à vous autres lecteurs, les conditions et le temps qu'il faut pour passer la mer de sable, même si de tout ce chemin j'ai peu à raconter sinon qu’on a marché. Cependant, pour ne rien négliger, je me dois de vous faire la description des conditions et du temps qu'il faut pour traverser ". Un peu plus loin, justifiant la description de la ville de Syène, il répète : " Je marchais par toute la ville, c'est pourquoi je suis forcé de vous en faire le plan et la description parce qu'il me semble que ce serait un tort ne le taire et ainsi je ne peux manquer de vous avoir tout dit $»^{10}$.

10 BAV, Barb. lat 4791, fol. 23v et 24 : « Non voglio manchare ad voi altri lettori di non dirvi il modo ettenpo che si passa il mare renoso con tutto che in questo camino nonarei addirvi altro che caminare ma per non lassare cosa arretro so forzato ad farvi la discritione il modo eltenpo che si passa [Description de la ville de Syène] chaminai tutta la città dove che qui di sotto so forzato farvi la pianta ella discritione per che mi pare che si faria torto attaciella et con questo io non posso manchare di non dirvi il tutto ". 
Il dit régulièrement qu'il n'est pas un savant, mais un praticien, comme si son lecteur était plus savant que lui. Prenons l'exemple de la description de Babylone, qu'il confond avec un faubourg du Caire, comme c'est souvent le cas dans les descriptions anciennes de la ville, mais au XVI ${ }^{\mathrm{e}}$ siècle cette confusion est levée et elle est d'autant plus étonnante que son voyage au Caire n'est pas fabuleux.

J'ai voulu voir toute chose par le menu pour vous en donner un rapport clair et complet (...) il faut que vous sachiez que je ne mets pas la moitié de ce que j'ai vu parce que cela me paraît une histoire longue et aussi pour laisser la possibilité de le vendre et de le faire circuler : si je mettais tout cela ferait un livre si gros qu'il fatiguerait bien des lecteurs, surtout à ceux à qui plaisent ces (récits de) voyages, les savants et les marcheurs (...) moi je suis dépourvu de savoir de quelque sorte que ce soit, ce que j'écris n'est que le fruit de la pratique, d'avoir vu et cheminé et ainsi je ne livre que des souvenirs, laissant le reste à qui le corrigera le mieux ${ }^{11}$.

Le lecteur n'est jamais identifié, il n'y a pas de dédicace, pas de destinataire explicite. Cependant, cet interlocuteur est posé comme italien, catholique (" latin » selon le terme de l'auteur) et connaissant différentes régions d'Italie. Giorgio fait en effet de nombreux parallèles dans ses descriptions avec des villes italiennes (Rome et Venise, en particulier). En dehors de la coquette distance de savoir posé entre un lecteur supposé savant et le "pratico " Giorgio, l'auteur se place dans une même communauté. Il appelle souvent une complicité en évoquant un parallèle : "Nous dirions en Italie..." ou tel rite "ressemble, dirions-nous nous autres ", au baptême, etc.

Lécriture de ses voyages, qui tiennent plus de la description du monde médiéval que du récit de voyage proprement dit, comme les récits autobiographiques où il ressasse de plusieurs manières son " origine " et ses tribulations de renégat, cherche constamment, tout en témoignant de contrées lointaines et d'expériences de la dissemblance, à réduire la distance qui sépare Giorgio de son lecteur. Il essaie de rapprocher le dissemblable et le lointain de lui de deux manières. La première est partagée par de nombreux voyageurs, consiste à établir sans cesse des comparaisons avec le monde du lecteur : en matière de climat, de botanique ou de coutumes, le renégat prend toujours le monde italien et catholique comme étalon. La deuxième, plus étrange, semble être de rapporter des clichés tout à fait éculés sur les lieux traversés, voire (comme dans le cas de Babylone, placée près du Caire) en reprenant des erreurs anciennes

11 Ibid., fol. 54 : "O volluto veder(e) ogni cosa minutamente per darvi chiaramente adguaglio del tutto [...] voi ave(te) di pensar(e) che no(n) metto lameta di quel che ovisto per che mi pare che sia una longa storia et anchora lo fo per dar(e) piu spaccio di spidimento per che mettendo cosa per chosa saria uno volume tanto grande che in crescieria amolti e massimo ad quelli che piacieno questi viaggi li scienti elli caminatori [...] io so privato di ogni sorta di scienzia q(ue) 1 che scrivo et solo praticha in avere visto et caminato et cosi ne fo solo un richordo riservando il resto ad chi corraggiera meglio". 
dénoncées par tous les voyageurs du $\mathrm{XVI}^{\mathrm{e}}$ siècle. Certes, en ce qui concerne la Chine, on peut imaginer que Giorgio ne s'y est pas rendu et qu'il ne puise pas aux sources les plus récentes ou les plus fiables pour la décrire. Mais il colporte autant de vieilles légendes sur les lieux qu'il a sûrement ou très probablement traversés. Cela est plus énigmatique.

Or, je pense que la réponse est peut-être à rechercher dans la communication de Sylvia Giocanti sur Montaigne ${ }^{12}$. L'estrangement délégitime les jugements. C'est pour Montaigne très positif. On peut penser que pour Giorgio c'est un cauchemar. Ce marginal doit tout faire pour ne pas apparaittre comme dissemblant, étrange, dissonant. L'estrangement, délégitimant, est un luxe qu'il ne peut pas se payer, en quelque sorte. Il lui faut se situer sur le terrain banal, les lieux communs parce que son but est l'intégration. Le monde ne branle pas du tout chez Giorgio, pourrait-on dire pour paraphraser Montaigne : en tout cas, il ne doit pas! D'autant moins qu'il cherche à se vendre comme le détenteur de vérités religieuses plus hautes. Il prétend connaitre mieux que quiconque le vrai en la matière, parce qu'il a éprouvé dans sa chair la différence des religions. Et là, dans ce seul domaine, il raconte des choses peu banales et tout à fait non conformes. L'hypothèse qui se dégage est que l'insistance de Giorgio à peindre un monde sans surprise pour son lecteur est un moyen d'obtenir un consensus sur fond duquel ses positions religieuses apparaissent moins dissemblables. Il fait référence à un savoir médiocre et périmé, mais à un savoir commun. Cela lui permet d'authentifier peut-être des positions religieuses tout sauf communes.

En effet, Giorgio aime à se montrer comme celui qui a vécu la différence religieuse de l'intérieur et donc est seul habilité à en parler sans mentir. La différence religieuse s'éprouve physiquement pour Giorgio : quand il était musulman, il était en excellente santé et vivait dans l'aisance. En revanche, si son corps ne connaissait pas la douleur, son âme était tourmentée par des accès de mélancolie et des cauchemars. L'idée du matérialisme de l'islam, dont le paradis réserve des plaisirs charnels, n'est pas du tout nouvelle dans la polémique chrétienne, et se trouve déjà au $\mathrm{X}^{\mathrm{e}}$ siècle chez Georges le Moine ${ }^{13}$, mais ce qui est intéressant c'est sa probation personnelle par Giorgio. Depuis qu'il est redevenu, définitivement, chrétien, son corps va très mal (il a souffert maladies, dommages matériels, pauvreté) mais son âme est en paix et il ne connaît ni insomnie ni maux de tête. Le choix du christianisme est dicté par cette expérience physique :

Je trouve que le bien de l'âme vaut mieux que le bien du corps, parce qu'il contente l'âme et le corps (...) En effet le corps est mélangé de divers métaux qui ne tiennent ensemble que grâce à l'âme, c'est pourquoi il faut contenter

12 Voir l'article ici publié de Sylvia Giocanti.

13 Voir Alain Ducellier, Le Miroir de l'Islam : musulmans et chrétiens d'Orient au Moyen Âge (VII XI siècles), Paris, Julliard, 1971, p. 208. 
d'abord l'âme. Qui veut se conduire autrement vit et meurt comme un chien ou un autre animal répugnant, parce que l'âme vient du ciel (...) et le corps ne peut demeurer sans l'âme ; aussi, au bout de mes mutations d'âmes et de corps, j'ai décidé de rester avec l'âme et que le corps aille où il veut ${ }^{14}$.

Finalement, le renégat relaps présente sa captivité comme un martyre et ses années en terre d'Islam comme une expérience unique qui lui a permis de mieux discerner la vraie foi.

Je suis persuadé que ma foi est la plus juste de toutes parce qu'elle a été conquise à la sueur du sang... cette Sainte foi bien peu la connaissent parce qu'il faut pour cela être patient et prendre le monde à rebrousse poil et attribuer le bien et le mal au Ciel (...) En vivant au milieu des Turcs, on apprend à mieux connaitre le Créateur ${ }^{15}$.

Souvent le récit de Giorgio est ainsi au bord de se transformer en apologie du catholicisme mais bizarrement, chaque fois qu'il met en scène la supériorité du christianisme « latin », c'est immédiatement pour plaider que " ognuno creda a suo modo " (que chacun croie à sa manière) et pour la concorde des religions (à l'exclusion le judaïsme), en insistant sans cesse sur le fait que l'islam et le christianisme latin sont extrêmement proches, tout juste séparés par le baptême...

Ces deux fois (le christianisme et l'islam), qui sont faites toutes les deux par le Ciel, devraient être amies et sont plus désunies que toutes les autres, à cause de notre orgueil. Les Tatares, les Scythes et les Indiens se contentent de ce que chacun croie à sa manière et entre eux ils ne réduisent personne en esclavage. Tandis que nous, insatiables, nous en venons toujours aux mains ${ }^{16}$.

Un épisode résume tout le propos de Giorgio, c'est celui du prétendu colloque de Salonique. Ce " concile sur le fait de la religion ", dont les nombreuses réunions entre catholiques et protestants ont dû lui donner l'idée, sans parler du concile de

14 BAV, Barb. lat 4791, fol. 155 : «Prouo per me che quando il corpo staua bono ellanima patiua di modo che trouo che meglio e auere ellanima bonna che il corpo perche contenta ellanima \& contento il corpo (...) il nostro corpo sta in pastato da diuersj metallj \& tuttj questj metallj non si possano vnire insieme senzia ellanima doue che di bisogno annoj per forza stare piu presto se suggietta \& contentare ellanima che il corpo chi uole alla fine conduciersj altrj mantj uiue $\&$ more in modo di vno cane oaltro animale bruto perche ellanima uiene dal cielo enon tiene fine $\&$ il corpo uiene dalla terra pieno di principio $\&$ corto del suo fine $\&$ questo lo dicho che il corpo non po stare senzia ellanima donde che nelle mie mutationj di corpo edianima misorisoluto stare collanima eil corpo uada doue si uoglia andare \& sie il uero di quanto one patito senpre».

15 Ibid., fol. 149 : «Vo persuendo che la fede mia sia la piu giusta di tutte perche \& conquistata per sudore di sangue (...) quaesta Santa fede pochi la cognoschano per che edi bisogno essere patiente \& pigliare il mondo per il uerso \& il male eil bene ad triburlo dal cielo (...) stando fralj turchi si cogniscie megli il creatore».

16 Ibid., fol. 317v-318: "perche trouo due fede che sofatte peruia del cielo che aueriano aessere amicj estanno piu dissonite che tutte lealtre questo ne causa lasoperbia nra hio uengho itartarj esciti \& indichj che si contentauano di ogniuno creda adsuo modo \& fra di loro non si faschiauo nissuno \& noj insaziabilj ad ognj hora simo alemanj ». 
Trente, se serait tenu sous l'égide du patriarche de Constantinople entre 1555 et 1557, en mettant en présence " quatre nations » : Grecs, Turcs, Juifs et Perses. Il n'y a bien sûr aucune trace d'une telle réunion. Après la chute de Constantinople, le patriarche œcuménique est souvent en visite dans les capitales provinciales pour récolter l'impôt et régler la vie de la communauté. Il y tient des " conciles ", mais où l'on ne débat pas du tout du dogme. Salonique est par ailleurs une ville où la communauté juive est très importante. Ces éléments ont pu donner l'idée à Giorgio de cette scène. Lui même y aurait été invité alors qu'il était en route vers la Macédoine pour une mission du sultan et aurait trouvé une assemblée " où l'on discutait sans aboutir depuis deux ans ». Il propose de mettre tout le monde d'accord sur un dessin. Il se recueille et dessine quatre palais, dont il livre une description très détaillée. Un seul est bien construit. Tout le monde reconnaît qu'un seul de ces palais est fait dans les règles de l'art. Giorgio révèle alors le sens de chaque partie de ce solide édifice, qui est, bien entendu, celui de la foi chrétienne latine. C'est un vieux « truc » de la joute théologique que de procéder par images et analogies. Pour notre auteur, qui dessine volontiers, il s'agit aussi, par l'image, d'éviter les pièges réducteurs de la parole et des définitions dogmatiques. D'ailleurs, alors qu'on pourrait s'attendre à ce que, triomphant, il conclut sur une reconnaissance de la supériorité du christianisme d'Occident, le colloque finit par " ordonner que chacun croit à sa manière " ${ }^{17}$ ! Il énumère les résultats concrets du rapprochement, qui sont essentiellement des accommodements sur les rites et la discipline du clergé. En ce qui concerne les Turcs, il « se sont changés de sorte qu'à part le baptême, ils sont comme chrétiens » et ils adoptent une "pragmatique ": outre les mesures discriminatoires contre les Juifs, il est beaucoup question de prendre des mesures pour alléger le sort des esclaves comme, par exemple, l'impossibilité de maintenir un homme plus de cinq ans en esclavage, ou encore la peine capitale pour le patron qui maltraite ses esclaves ${ }^{18}$. Nous voilà dans la fable pure, et c'est la fin des manuscrits, aussi bien de Sienne que du Vatican. Après, il y a seulement un vocabulaire multilingue italien, grec et turc et quelques copies de lettres. Mais c'est aussi le passage qui révèle le mieux la position et l'ambition de l'auteur.

C'est le dernier mot de Giorgio, qui a passé bien des jours en prison ou en esclavage, toujours accusé d'être un autre. Redonner vie à ce personnage, reconstituer sa culture et ses convictions, ses interactions avec les réalités italiennes, maghrébines ou turques auxquelles il a dû se confronter, ou encore

17 Ibid., fol. 325v : «Cosi fatto lesopra ditte figure leapresentaj nelloro concilio in modo che nissuno di loro potevano sapere che fede era (?) di chi sapeua essere doue che fra di loro giudico che il primo che era quello di tante colonne tutte uoleuano che fusse il piu forte edi piu durata \& cosj come loro giudicorno \& restatj dacordo hio (?) li chiamattj eddissilj il parte per parte \& cosi con questa mia figura si finj questo tale concilio con ordinare che ognj vno creda adsuo modo ».

Ibid., fol. 326. 
le rôle de l'écrit dans ses stratégies de survie et d'intégration, sont des tâches minutieuses et qui peuvent sembler vaines pour un si modeste acteur. Le travail de Carlo Ginzburg, constitue - non seulement un encouragement à cette entreprise ! - mais aussi offre une méthodologie pour exploiter au mieux les traces laissées par des personnages au sens propre excentriques.

Florence Buttay EA 2958 CEMMC

Université Michel de Montaigne Bordeaux 3 Florence.Buttay@u-bordeaux3.fr

\title{
Résumé
}

La notion d'" estrangement " trouve un écho particulier dans l'étude des écrits des voyageurs. Surtout quand, comme dans le cas de Giorgio del Giglio (1507 ?-1579 ?), ces écrits devaient à la fois rendre familier à ses lecteurs, Italiens et catholiques, les contrées décrites, mais aussi chercher à réduire la distance qui le séparait d'eux. En effet, d'origine marrane probablement, plusieurs fois converti à l'Islam et tenté par le calvinisme, Giorgio écrit pour trouver sa place dans l'Italie de la deuxième moitié du XVI ${ }^{\mathrm{e}}$ siècle où ses convictions iréniques ne sont plus guère de saison.

\section{Mots-clés}

Récits de voyages, $\mathrm{XVI}^{\mathrm{e}}$ siècle, Italie, Méditerranée, frontières religieuses et culturelles.

\begin{abstract}
The notion of "estrangement" fits particularly well to the study of travellers'books. Especially when, like in case of Giorgio del Giglio (1507?-1579?), these books were supposed not only to make familiar to his italian catholic audience the lands he described, but also to reduce the distance between him and his readers. Coming from a Spanish family of Marranos who immigrated to Tuscany, renegade of both Muslim and Roman Christianity faiths, Giorgio writes in order to find a place in the italian society of the second half of the Cinquecento, when his irenic beliefs were dangerous to expose.
\end{abstract}

\section{Keywords}

Travellers'books, XVI th century, Italy, Mediterranean Sea, religious and cultural boundaries. 